\title{
Learning by the experience of others: an experiment on information contagion
}

\author{
Alessandro Narduzzo ${ }^{1}$ and Massimo Warglien ${ }^{2}$
}

1. Dipartimento di Economia e Studi Aziendali, Università di Bologna, and Experimental Economics Lab, Università di Trento.

2. Dipartimento di Economia e Direzione Aziendale, Università di Venezia, and Experimental Economics Lab, Università di Trento. For correspondence: warglien@unive.it 


\begin{abstract}
Advances in stochastic system analysis have opened the way to a reconsideration of the processes through which behaviors spread in a population of individuals or organizations. One peculiar phenomenon affecting diffusion is information contagion (Arthur and Lane 1994). When agents have to choose on the basis of other people's experience, rather than relying on their own direct observations, information externalities arise that drive towards the emergence of the arbitrary, stable dominance of one product over the competing one. We reproduced in controlled laboratory conditions the process of information contagion. The experiments show that when agents can only resort to the observation of other people's experience in choosing between competing alternatives, the choice process generates some peculiar features:

- information contagion among subjects generates self-reinforcing dynamics, amplifying initial asymmetries of products' market shares;

- this in turn produces path-dependent trajectories, highly dependent on early events in the choice sequence;

- arbitrary asymmetric market shares tend to be stable in the long run, exhibiting lock-in phenomena;

- agents choice criteria are heterogenous, giving rise to a mix of positive and negative feedback in the choice process, with the mix and the timing of such criteria affecting the final outcome.
\end{abstract}




\section{Introduction}

Learning by the experience of others is a central feature of most cognitive and choice activities, both in individuals and organizations (Levitt and March 1988). It is at the very heart of phenomena such as cultural transmission, skills acquisition and diffusion processes. Nevertheless, the study of how people use other people's experience is a rather underdeveloped area of the analysis of economic behavior, often reduced to simplistic assumptions about pedestrian imitation or epidemiologic contagion.

Advances in stochastic systems dynamics (Hill et al. 1980) have opened the way to a reconsideration of the mechanisms through which behaviors spread in a population of individuals or organizations, accounting for a wide set of diffusion processes without needing to hypotesize brute imitation among agents. In facts, it has been shown that even quite sophisticated decision rules can generate dynamics that may look at a first glance as an outcome of simple mimetic behavior.

One example is Brian Arthur's analysis of externalities and technology diffusion, relying on generalized urn schemes (Arthur, Ermoliev and Kaniovsky 1987). Arthur has shown that, in presence of externalities "a technology that by chance gains an early lead in adoption may eventually "corner the market' of potential adopters, with the other technologies becoming locked-out." (Arthur 1989, p.116). Thus, even sub-optimal technologies can dominate the market when there are increasing returns, due for example to external economies of scale, learning by doing, product compatibility. Moreover, when such conditions hold, early random historical accidents determine arbitrary but stable equilibria. A well-known historical exemplification of these processes is the emergence of QWERTY as a dominant keyboard design (David 1985). Although one might consider the long run effects of early adopters' choices as a consequence of imitative behavior by late adopters, Arthur's analysis shows how these seemingly mimetic dynamics are generated by rational choice in presence of externalities.

An even more compelling example has been given by Arthur and David Lane's Information Contagion model (Arthur and Lane 1993). In this model, Bayesian agents have to decide whether to buy a product A or B relying only on the observed performance obtained from the product by a sample of previous adopters. The model shows that, even in the absence of usual externalities, Bayesian agents will be prone to arbitrarily privilege one of two equally attractive technologies, when their choices will have to rely on the observation of previous adopters' experience rather than on their own direct one (in other words, when sampling is on other agents rather than on products). The information contagion effect is based on the fact that, if agents can only sample previous adopters' experience with a product, the single product that has been more chosen in the past has a larger probability to be in the agent's sample. Imagine that you want to buy an expert system for motorcycle maintenance, and trying directly the competing products on the market would be too costly and long. Thus, you decide to sample ten motorcyclists that have already made a choice. Before you $80 \%$ of the buyers have already chosen Mechanisoft, while only $20 \%$ have chosen turboZen. If you ask your ten informants, probably a large majority of them will be a Mechanisoft user. Traditional models of imitation would predict that you will buy Mechanisoft according to the majority choice. Arthur and Lane have convincingly explained that there is no need to hypothesize such a trivially conformistic behavior to explain the choice contagion. It is rather the fact that you have more information on Mechanisoft that will lead you (if you are not 
risk prone) to adopt it. Provided that there is not too high a difference between the two products' average performances, if you are a risk averse Bayesian decision maker, it will be appropriate to privilege the product on which you have more information, and thus lower sample variance. Again, the model predicts that early adoptions will determine which product will finally dominate the market. The model also predicts that, in general, dominance will not imply monopoly: some diversity in the demand composition will anyway be preserved.

Dosi, Ermoliev and Kaniovsky (1994) have provided a complementary analysis, belonging to the same family of stochastic models. Their analysis is in a way cruder than Arthur and Lane's one, since positive feedback is directly provided by basic imitative behavior; on the other side, their model allows for considering heterogeneity in agents' behavior. As in the Information Contagion scheme, it is assumed that agents choose only on the basis of the observation of a sample of previous adopters. However, agents do not consider the experienced performance of the products, but only which product has been chosen by their informants. The model assumes that agents will follow the majority of informants with probability $\alpha$ and will make the "minority choice" with probability 1- $\alpha$. The results are close to Arthur and Lane's ones: in their model, there are two products with equal but unknown utility. If $\alpha<1$ and the sample size $>1$, asymptotically arbitrary but stable market shares will emerge, with the exclusion of monopolistic outcomes (thus, there will be an equilibrium coexistence of variety despite positive feedback factors).

Under the guidance of such literature, we have tried to reproduce experimentally choice processes in which a population of agents faces binary choice between two alternative products of equal utility, unknown to agents, relying only on information provided by a sample of previous adopters. The experiments show that information contagion arises generating arbitrary stable dominance of one product according to what predicted by the Arthur and Lane's model. A closer analysis of subjects' choice heuristics shows significant heterogeneity among actors, thus supporting the assumptions and some conclusions of the Dosi, Ermoliev and Kaniovsky's paper.

\section{The experimental design}

We ran two blocks of experiments. The first experimental series was aimed at testing the emergence of the arbitrary dominance of one out of two equally attractive alternative products, under the conditions of an information contagion model. The outcomes of the first experimental series have induced us to conceive a second block of experiments, in order to better explore the stability of emergent equilibria.

The structure of the first experimental series is quite simple and straightforward. It implies a sequence of individuals choosing between two competing products on the ground of information obtained from agent-based sampling, i.e. from a random sample of agents having already chosen between the two products. Each subject obtains from sampled agents only two pieces of information: the product the agent has chosen, and the utility she has extracted from it. The utility of each product is represented by a performance index, expressed by a scalar. The performance is a random variable, with average and standard deviation unknown to the experimental subjects. When a subject chooses a product, she is randomly assigned a performance index. In our experiment, the two competing products have the same utility, i.e. their performance index has the same average 
and the same standard deviation. As subjects experience a product's utility, their product choice and the related performance index are recorded in a data base from which the subsequent subjects can draw their own sample of informants.

In more detail, a single baseline experiment runs as follows. About 50 subjects are sequentially called to participate to the experiment. During the experiment, things are arranged so that they cannot communicate to each other. They are provided with an instruction sheet, explaining that they have to choose between two alternative products (A and B), whose "objective" performance is expressed by a number and is uncertain. They are also explicitly informed that a same product can generate different performances with different individuals. They are told they can only obtain information about the products from a random sample of six subjects, chosen among those having already made the choice.After reading the instruction sheet, subjects reach a computer whose screen presents information as in the following example (Fig. 1).

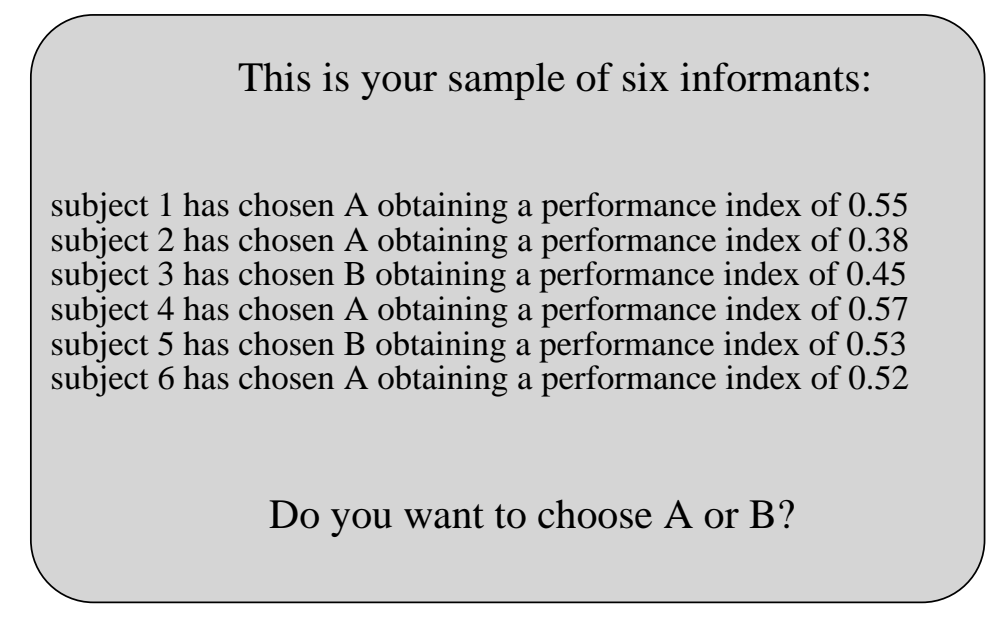

Fig. 1

Subjects are asked to type in the keyboard which product they choose, given the information available. As they make the choice, they obtain from the screen their own numeric performance index (Fig. 2). The index is a random number drawn in the interval [0.25..0.75], with a rectangular distribution.

You have chosen A

Your performance index is: 0.47

Fig. 2

Immediately after completing this first part, the experimental design prescribes that each subject has to deal with a second part of it. A new information sheet tells him that thanks to a technological innovation, two new products, $\mathrm{C}$ and D, have been introduced. Subjects are told that these products have superior performance over A and B (although no more information is given about their performance). Subjects have to choose between C and D. Again, the screen of a second computer exhibits a sample of six informants' choices and performances. Remark that, again, the two 
products have identical performance, drawn from a rectangular distribution in the interval [0.60..1.00].

To complete the first experimental block, we have designed a control experiment aimed at comparing the dynamics generated by learning from the experience of others with the dynamics generated by direct experiential learning. Thus, in this control experiment, we have allowed subjects to perform product-base sampling. In this case, subjects can try one or both products up to six trials, and observe the performance obtained in each trial; then, they choose. In other respects, the structure of the experiment is identical to the baseline experiment.

The second experimental block is a simple variant of the first one with only two design changes. The first and more important change is that the experiment length is protracted from a sequence of about 50 subjects to a sequence of more than 170 subjects in order to see whether the arbitrary dominance effects are stable. A second minor change is to express the performance of the two products using integer numbers between 0 and 99 rather that real numbers in the [0..1] interval, in order to provide a more natural numerical representation of the performance ${ }^{1}$.

\section{Experimental results ${ }^{2}$}

In the first block there were three runs based on the information contagion scheme. Each run included two choice situations (A vs. B and C vs. D). In most runs very neat path dependent dynamics emerge. We have been looking at cumulative market share for each product over the whole sequence of individual choice. Four aggregate choice dynamics out of six generated a remarkable and stable prevalence of one product over the other one.

Table 1: Baseline experiment

\begin{tabular}{|l|l|l|l|l|l|l|l|l|}
\hline Baseline experiment & \multicolumn{1}{|c|}{$\begin{array}{c}1 \\
\text { A vs. B }\end{array}$} & $\begin{array}{c}1 \\
\text { C vs. D }\end{array}$ & $\begin{array}{c}2 \\
\text { A vs. B }\end{array}$ & $\begin{array}{c}2 \\
\text { C vs. D }\end{array}$ & $\begin{array}{c}3 \\
\text { A vs. B }\end{array}$ & $\begin{array}{c}3 \\
\text { C vs. D }\end{array}$ & $\begin{array}{c}4 \\
\text { A vs. B }\end{array}$ & $\begin{array}{c}4 \\
\text { C vs. D }\end{array}$ \\
\hline \hline length (n. of subjects) & 64 & 64 & 30 & 30 & 36 & 36 & 54 & 54 \\
\hline final A\% (share) & 52 & 30 & 20 & 23 & 72 & 39 & 55 & 65 \\
\hline final B\% (share) & 48 & 70 & 80 & 77 & 28 & 61 & 45 & 35 \\
\hline
\end{tabular}

Notice that sometimes A dominates B and C dominates D, while sometimes the opposite happens, despite the fact that the initial conditions of the process are always the same in the two situations.

1. Lane's suggestion

2. The subjects were students in Economics or Management courses at the University Ca' Foscari of Venice. 


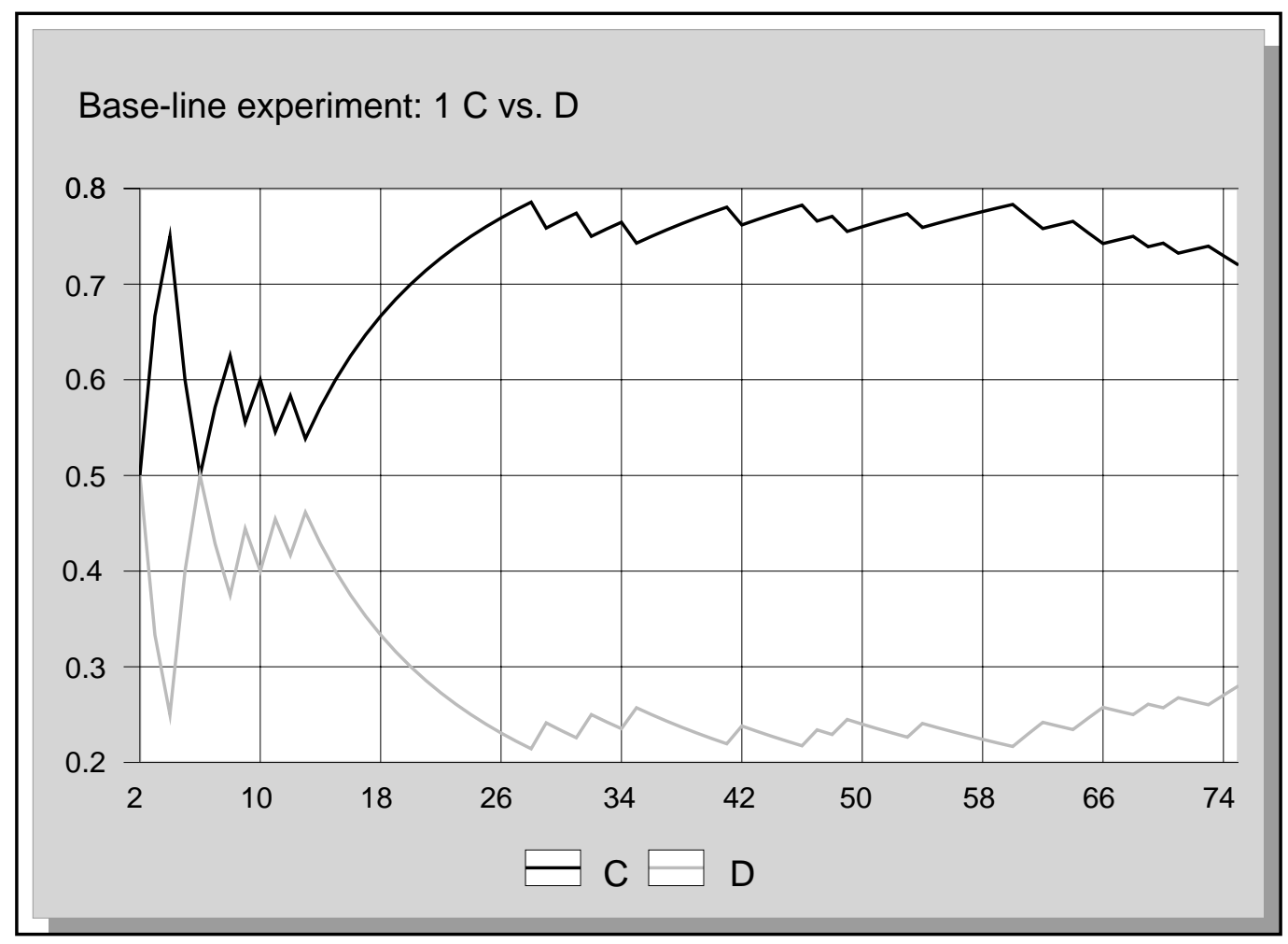

Fig. 3

However, the most interesting information are those concerning the trajectories of market shares. Fig.3 shows a typical path in a $\mathrm{C}$ vs. D run. The reader familiar with urn scheme will recognize a behavior analog to that of a Polya's urn. Early fluctuations give rise to a seemingly stable configuration, with product $\mathrm{C}$ taking over around $75 \%$ of the "market share". Two other cases, however, show a different path. In one case, an initial, long phase of oscillations around the $50 \%-50 \%$ partition, is followed by what might be (but need not necessarily to be) the beginning of a divergence of market shares. A second case (the most troublesome) shows an initial divergence reabsorbed and a quick comeback of the 50-50 shares.

Despite those two puzzling results that defer to the following exploration of longer time series, the first round of the experiment supports the Arthur-Lane claim that strong informational advantages can arise from early accidental choice events.

The control experiment, where subjects can make experience of alternatives before choosing, provides another source of support to the Information Contagion hypothesis. 
Table 2: Control experiment

\begin{tabular}{|l|l|l|}
\hline \multicolumn{1}{|c|}{ Control experiment } & \multicolumn{1}{c|}{$\begin{array}{c}1 \\
\text { A vs. B }\end{array}$} & $\begin{array}{c}1 \\
\text { C vs. D }\end{array}$ \\
\hline \hline length (n. of subjects) & 40 & 40 \\
\hline final A\% & 55 & 45 \\
\hline final B\% & 45 & 55 \\
\hline
\end{tabular}

The Table 2 and the figure neatly show the working of the law of large numbers. As the number of choices cumulates, the market shares of the two products converge towards a 50\%-50\% configuration.

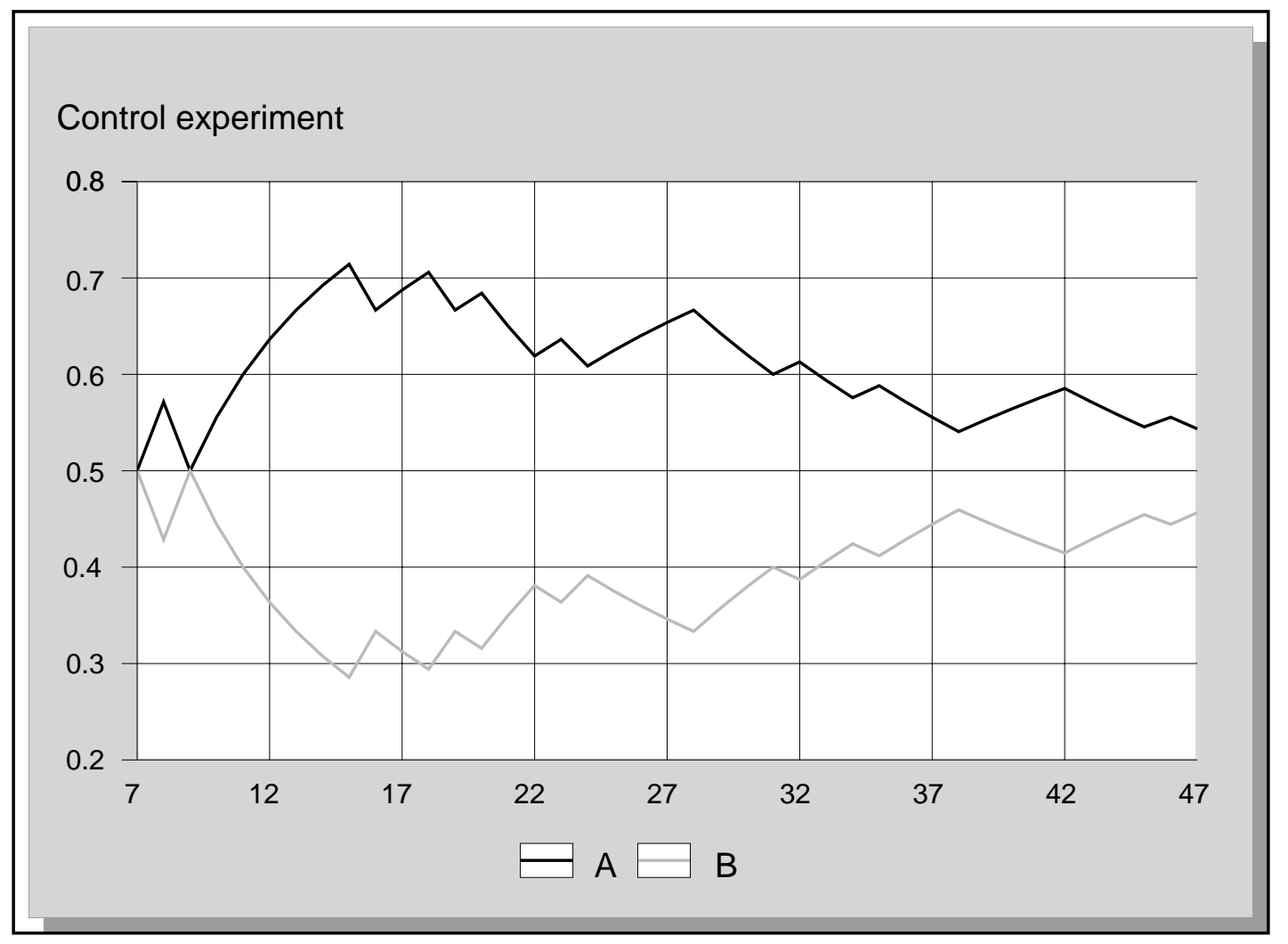

Fig. 4

The first experimental round left us with a serious question about a stability of the asymmetric market shares emerging from the choice sequences. As reported above, the second round was designed to provide some insights in the longer run behavior of the process. Therefore we per- 
formed a second round of three additional experimental runs following the scheme reported in the session two.

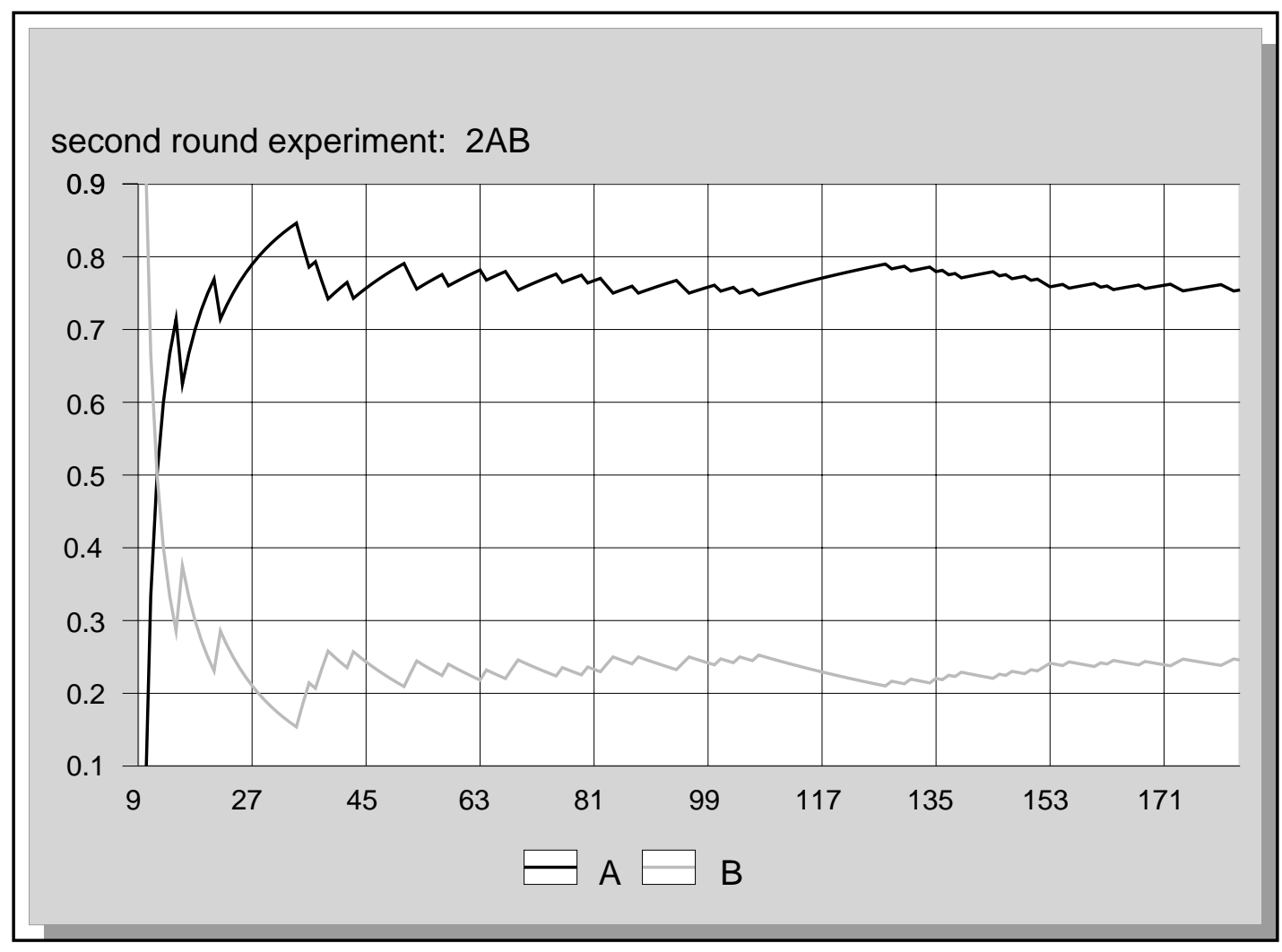

Fig. 5

Table 3: Baseline experiment - 2nd block

\begin{tabular}{|l|l|l|l|l|l|l|}
\hline \multicolumn{1}{|c|}{ Baseline experiment } & \multicolumn{1}{c|}{$\begin{array}{c}1 \\
\text { A vs. B }\end{array}$} & $\begin{array}{c}1 \\
\text { C vs. D }\end{array}$ & $\begin{array}{c}2 \\
\text { A vs. B }\end{array}$ & $\begin{array}{c}2 \\
\text { C vs. D }\end{array}$ & $\begin{array}{c}3 \\
\text { A vs. B }\end{array}$ & $\begin{array}{c}3 \\
\text { C vs. D }\end{array}$ \\
\hline \hline length (n. of subjects & 176 & 176 & 175 & 254 & 186 & 186 \\
\hline final A\% (share) & 56 & 58 & 75 & 57 & 41 & 40 \\
\hline final B\% (share) & 44 & 42 & 25 & 43 & 59 & 60 \\
\hline average As\% on last 100 & 55 & 58 & 76 & 58 & 41 & 35 \\
\hline std dev on last 100 & 0.01 & 0.01 & 0.011 & 0.008 & 0.011 & 0.027 \\
\hline
\end{tabular}

a. This series is made by new 78 subjects' choice appended to the series 1CD (See Note 1 on this page). 
Table 3 reports the results of this second block (unfortunately the results of a $\mathrm{C}$ vs. D run were lost). Together with the cumulative market share at the end of the experiment, we also report the average and the standard deviation of the cumulative market share over the last 100 choices. One can easily see how stable is the behavior of such variable as the choice sequences go beyond the early period. Fig.5 pictures one run of the experiment, offering graphical evidence for the same findings. Although more rigorous inference is needed in order to better understand the nature of such trajectories, we claim that the hypothesis of a lock-in of market shares can be supported on the basis of such data.

To find some hints about choice heuristics that people used during the experiment, we conduced some interviews with the experimental subjects immediately after their choice, and we also performed some "thinking-aloud" protocol analysis (Ericsson and Simon1984) with a separate set of subjects. Despite the obvious limitations of such methodologies in order to capture how subjects really decide, we could find clues suggesting that subjects used a variety of individual rules of thumb. Not surprisingly, only one of these can be reconciled (and only under special conditions) with the bayesian optimization criteria assumed in the Arthur-Lane paper. Although individual subjects report their decision rules in a variety of ways, most criteria can be referred to four basic choice heuristics, that reflect behaviors well known in the psychological literature on decision making. Notice that all subjects, being students in management and economics, had prior training on probability and optimization techniques.

The mean rule. Subjects choose the product which shows the higher mean in the sample. This is the only rule to be compatible with bayesian optimization, in the limit case of non informative prior distribution and risk-neutrality of subjects. And it is the simplest application of our subjects' statistical training (the only case in which such training explicitly surfaces, by the way).

The min rule. Subjects choose the product with the higher minimum performance in the sample. This rule substantially resembles to the maximin rule introduced for the first time by the statistician Abraham Wald. It is the expression of an extremely prudential attitude. Subjects' first concern is to avoid bad outcome, as far as possible. They prefer to maximize their security rather than to try to maximize their reward (Colman 1982).

This rule reflects an extreme form of loss aversion, if loss is defined as the difference between the average (anchoring) performance in the sample and the actual single performance.

The max rule. Subjects choose the product which shows the highest performance in the sample. This procedure is especially intriguing, since it seems to have little rational motivation. Thus it is interesting to look at what people say while using this rule. The most frequent attitude is well exemplified by the following exerpt: "well, let's see... the best outcome is that one... I should be able to get the same performance.. if the smartest guy could do it, I should too". Moreover, our subjects spontaneously used to guess some reasons to justify low performances referred by other informants in their sample; they supposed that those informants were unskilled and that low outcomes were due to negligence, wrong expectations, inappropriate utilization of the product. This decision rule, that gives too credit to a positive information in the sample and overweights the value of a single experience, seems to reflect a well known bias in human decision making, the "illusion of control" (Langer 1975). When confronting a task which implies uncertainty, people often have expectations of personal success much higher than the available information should 
allow to. This expectancy of a personal success is generally due to skill-related factors, such as familiarity with the problem, choice, competition, involvement, etc. "When a chance situation mimics a skill situation, people behave as if they have control over the uncontrollable event when the fact of success or failure depends on chance is salient " (Langer 1975). This happens not only in tasks where personal ability can matter, but also in pure chance gambles like in lotteries (Langer and Roth 1975).

It has been observed that this rule is often used by voters in elections and by naive decision-makers (Colman 1982). March and Shapira have shown how the illusion of control is a fundamental component of the managerial ideology of risk taking (March and Shapira 1987). Managers generally consider uncertainty as manageable; they consider as part of their role getting the best outcome in a risky task. They do not consider risk in probabilistic terms, but rather expect to "reduce the danger while retaining the gain" (p. 1410). Clearly, most of our subjects resorting to the max rule incur in the illusion of control bias, and they probably draw on the same social view of risk taking managers do.

The popularity rule. Subjects choose the product that most informants in their sample have already adopted. This is an ambiguous rule, that may be supported by very different motivations. On the one hand, there are the well-known pressures towards social conformity that explain in many circumstances mimetic behavior. On the other one hand, there may be more rational motivations for following the majority. People can trust the rationality of others, and thus assume that "if the majority of them did it, they had to have good reasons for it"; otherwise, especially when the majority is large and the difference between the two products' sample mean is small, people can be motivated by risk aversion.

These heuristics are not necessarily used in insulation. When the first one tried by a subject fails to provide a clear guidance to the choice, she often tries sequentially another one - for example, if the sample means of the two products are too close, people may look at the most popular product. Therefore, it may happen that the heuristic that is actually used by a subject is not the first one that came in her mind; rather, the applied rule is the first one that makes sense with the information sample. The following excerpt, picked out from one of our protocols, offers another example of such a phenomenon.

"Which informant should I trust?...Mmm, maybe I should calculate a mean of the performances. Well, 50.. 80.. 50. 150 over 3...60 for B and ....60 for A. Ah! They do have the same mean! Therefore, basing on the average I do not get any information to decide...The best outcome for product $\mathrm{A}$ is 80 , while the best one for B is $70 \ldots$ I choose A."

Moreover, rules are not stable. When people move from the choice between A and B to the choice between $\mathrm{C}$ and $\mathrm{D}$, they sometimes abandon the rule used in the first part of the experiment and try a new one. Interviews suggest that this may often be related to disappointment with the first experience. This may lead to ask whether some heuristics are more disappointing than others - a line of inquiry that we plan to pursue as a further development of this experiment. On the other side, change in rules may be related to context-sensitivity of the rules themselves. For example, different samples of observations might induce different rules in the same subject. Again, we see exploration of context-sensitivity of rules as a major further development of our current work. These different individual decision rules have different implications when aggregated in a sequence of choices. Some of these rules do generate positive feedback, reproducing information contagion 
dynamics, while others do not. Quite surprisingly, Lane and Vescovini (this issue) have shown that the aggregation effects of such heuristics run against naive intuition. For each of the heuristics reported by our subjects, Lane and Vescovini analyzed the dynamics of market shares, under the condition that all the adoptants use the same rule of thumb. While the mean rule and the min rule do never produce path dependent scenari given our experimental conditions (products with identical characteristics and six-informant samples), the popularity rule always generates path dependent market shares. Finally, the max rule originates a variety of different and stable market configurations.

An implication of these differences among rules is that the emergence of path dependence and information contagion is thus related to the mix of rules actually used in each experiment. The relevance of the sorting of choice heuristics is shown by the following example. In two different experimental runs, the same random number generator seed was employed to govern sampling and attribute a performance index to each individual choice. Thus, identical behaviors should have led to the same path. But exactly the opposite has happened: while in the first experimental run C has taken about $75 \%$ of the market share, in the second run D has taken an $80 \%$ share!

\section{Summary and further improvements}

The results of our experiments are easily summarized. We have shown that when agents can only resort to the observation of other people's experience in choosing between competing alternatives, the choice process generates some peculiar features:

- information contagion among subjects generates self-reinforcing dynamics, amplifying initial asymmetries of products' market shares;

- this produces path-dependent trajectories, highly dependent on early events in the choice sequence;

- arbitrarily asymmetric market shares tend to be stable in the long run, exhibiting lock-in phenomena;

- agents choice criteria are heterogenous, giving rise to a mix of positive and negative feedback in the choice process, with the mix and the timing of such criteria affecting the final outcome.

We see possible developments of the experiment in two main directions, one allowing a deeper understanding of individual behavior in information contagion processes, the other one related to the aggregation outcomes when other forms of agent differentiation are introduced.

At the individual choice level, a central question is whether differences in individual decision rules simply reflect the subjects' cognitive history prior to the experiment or whether these differences can be related to the process of information contagion itself. A first major issue concerns rule learning: how do people change rules after making experience of their outcomes? Are there more disappointing rules, so that we can expect the rule mix to change in predictable ways as a result of choice experience? A second issue is related to the context-sensitivity of heuristics. Do people tend to use different rules when confronted with different sample information? As the unfolding of information contagion affects the urn from which samples are drawn, how do those endogenous changes in the urn composition affect the mix of rules? 
At the aggregation level, our experimental design provides a flexible building block for other experiments in order to expand the exploration of the information contagion under different conditions. A first extension is stimulated by the Lane-Vescovini paper, that suggests to investigate social efficiency issues when competing products have different utility. A second extension concerns the effect of space on contagion dynamics. One natural way to do it is to make the sampling of previous adopters dependent on their distance from the choosing subject, after having initially assigned a spatial allocation of experimental subjects.

Finally, an interesting question concerns the impact of differential access to experience in different subsets of the agents" population; for example, one might consider an "élite" group who has access both to direct and other people's experiences, and a "mass" with only access to indirect experience $^{1}$.

We expect these enrichments of the original experimental scheme to let emerge more differentiated behaviors, both at the individual and at the aggregate level, allowing us to reproduce a more articulate picture of how we learn from other people.

\section{References}

Arthur, B.W., 1989, Competing technologies, increasing returns, and lock-in by historical events, Economic Journal, 99, 116-131.

Arthur, B.W., Ermoliev, Yu.M., Kaniovsky, Yu.M., 1987, Path dependent processes and the emergence of macro-structure, European Journal of Operational Research, 1, 294-303.

Arthur, B.W., Lane, D.A., 1993, Information constriction and information contagion, Structural Change and Economic Dynamics.

Colman, A.M., 1982, Game Theory and Experimental Games, Pergamon Press.

David, P.A., 1985, Clio and the economics of QWERTY, American Economic Review, Paper and Proceedings, 75, 332-337.

Dosi, G., Ermoliev, Y., Kaniovsky, Y., 1994, Generalized urn schemes and technological dynamics, Journal of Mathematical Economics, 23, 1-19.

Ericsson, K.A., Simon, H.A., 1984, Protocol Analysis, The MIT Press.

Hill, B.M., Lane, D., Sudderth, W., 1980, A strong law for some generalized urn processes, The Annal of Probability, 2, 214-226.

March, J.G., Shapira, Z., 1987, Managerial perspectives on risk and risk taking, Management Science, 11, 1404-1418.

Langer, E.J., 1975, The illusion of control, The Journal of Personality and Social Psychology, 32, 311-328.

Langer, E.J., Roth, J., 1975, Heads I win, tails it's chance: The illusion of control as a function of the sequence of outcomes in a purely chance task, Journal of Personality and Social Psychology, 32, 951-955.

Levitt B., March, J.G., 1988, Organizational learning, Annual Review of Sociology.

Pirsig, R., 1974, Zen and the art of maintenance of motorcycle, Morrow.

1. We are grateful to Michael Cohen for this suggestion. 